\title{
Open-dulo: pêndulo invertido como plataforma educacional
}

\author{
Wagner Ferreira Andrade* Paulo Henrique dos Santos* \\ Alexandre Magno de S. Thiago Filho* \\ Nilton Costa Pereira de S. Thiago Neto ${ }^{* *}$ Gabriel Garcia* \\ * Departamento de Engenharia de Controle e Automação (DECAT) \\ Escola de Minas - Universidade Federal de Ouro Preto - UFOP \\ Ouro Preto, Minas Gerais, Brasil (e-mail: \\ wagner.andrade@aluno.ufop.edu.brpaulo.hs@aluno.ufop.edu.br \\ alexandre.filho@aluno.ufop.edu.br) \\ ** Departamento de Engenharia Mecânica (DEMEC) \\ Escola de Minas - Universidade Federal de Ouro Preto - UFOP \\ Ouro Preto, Minas Gerais, Brasil (e-mail: nilton.neto@aluno.ufop.edu.br)
}

\begin{abstract}
The inverted pendulum is a classic problem in the literature for the implementation and study of control techniques, which consists of balancing the body of a robot over two wheels. This paper proposes the creation of an open platform for teaching control, with low cost and high flexibility to implement different control strategies to achieve the balance of an inverted pendulum. The project allows students to apply embedded systems programming techniques, real-time systems, control theory and electronics. During the creation of the platform, the structure was designed over a printed circuit board to reduce the gap in the robot body. Two inertial measurement units were used, which are combined by a complementary filter for better reading of the variables (angular velocity and acceleration). We present the hardware development and the algorithm developed for validation of the concept, which was able to balance the robot during the tests.

Resumo: O pêndulo invertido é um problema clássico da literatura para implementação e estudo de técnicas de controle, o qual consiste em equilibrar o corpo de um robô sobre duas rodas. Este artigo propõe a criação de uma plataforma aberta para o ensino de controle, com baixo custo e alta flexibilidade para implementação de diferentes estratégias de controle para realizar o equilíbrio de um pêndulo invertido. O projeto permite ao aluno aplicar técnicas de programação de sistemas embarcados, sistemas de tempo real, teoria de controle e eletrônica. Atentou-se durante a criação da plataforma, projetar uma estrutura na própria placa de circuito impresso, para a redução de folgas no corpo do robô. Foram utilizadas duas unidades de medição inercial, que são combinadas por filtro complementar para melhor leitura das variáveis (velocidade angular e aceleração). Este artigo apresenta o desenvolvimento do hardware e do algoritmo desenvolvido para validação do conceito, o qual foi capaz de equilibrar o robô durante os ensaios.
\end{abstract}

Keywords: Inverted pendulum, Segway, Teaching platform, Real time, Control Palavras-chaves: Pêndulo invertido, Segway, Plataforma de Ensino, Controle, Tempo real.

\section{INTRODUÇÃO}

O desenvolvimento prático de conceitos ministrados em sala de aula torna o aprendizado mais eficaz, despertando no aluno maior interesse e motivação. De acordo com Soriano et al. (2014), até recentemente, kits didáticos voltados para tarefas práticas em ambientes educacionais eram produzidos por empresas que mantinham o hardware e software dos projetos fechados, resultando em plataformas de alto custo, pequena flexibilidade e de difícil interação com produtos e linguagens de programação de terceiros.

Em virtude das limitações impostas por plataformas de ensino comerciais, observou-se o crescente desenvolvimento de plataformas abertas (open source) nos últimos anos. Segundo Tuomi (2013), a rápida expansão do número de iniciativas de recursos educacionais abertos e os milhões de alunos que elas atraem pode facilmente ser interpretada como um indicador da revolução na educação e aprendizado.

Com o impulsionamento de tecnologias de baixo custo para prototipagem rápida, abriu-se um mundo de possibilidades para elaboração de projetos. Permitiu-se a concepção física de ideias, pesquisas e trabalhos que antes ficavam presos a simulação. $\mathrm{O}$ advento de equipamentos de baixo custo, como impressoras 3D, fresadoras CNCs, máquinas de corte a laser e kits de prototipagem alavancaram o desenvolvimento tecnológico da última década.

A comercialização de placas de desenvolvimento microprocessadas, como Arduino, Raspberry PI, ESP e MSP também contribuíram para a expansão do ensino. Como abordado por Jamieson and Herdtner (2015), essas plataformas acompanham, principalmente, três abordagens: aprendizado baseado em projetos, em que o aluno se envolve com tarefas e desafios especí- 
ficos para desenvolvimento do mesmo; projetos open source, os quais promovem a colaboração coletiva com o intuito de acelerar e aumentar a qualidade do projeto; e placas eletrônicas de prototipação onde o aluno realiza o desenho de placas de circuito impresso e as confeccionam para a solução do problema.

A demanda por capacitação tecnológica nas profissões tende apenas a aumentar. Segundo Vega and Cañas (2018), incorporar o ensino de robótica desde o nível fundamental é importante para o desenvolvimento de aptidões e afinidade de alunos com ciência, tecnologia, engenharia e matemática (STEM).

Diante da necessidade do contato entre estudante e plataforma de ensino, destaca-se sempre a importância das plataformas físicas de ensino, em contrapartida a simulações. Para tal, fazse necessário desenvolver plataformas robóticas de arquitetura aberta de baixo custo, replicáveis, simples e de alta adaptabilidade.

Plataformas de ensino de controle e robótica geralmente são baseadas em modelos bem conhecidos e estudados. Dentre eles destaca-se o robô do tipo Segway - um robô equilibrista de pêndulo invertido - que possui um corpo vertical equilibrado sobre duas rodas. Uma plataforma de ensino baseada no Segway foi apresentada por Nawawi et al. (2008) e teve por objetivo construir um Segway especificando o hardware, constantes físicas do modelo do robô e técnicas de controle aplicadas. Esta plataforma não foi idealizada para ser aberta e não possui seus arquivos de projeto disponíveis, tornando a replicabilidade inviável.

Outra plataforma de ensino baseada no Segway é a apresentada por Howard and Bushnell (2015). Assim como no trabalho anterior, é abordado o modelo do robô e suas constantes. Além disso, são aplicadas diferentes técnicas de controle para a validação do modelo e o sistema. Esta plataforma, diferente da anterior, é aberta, possuindo os dados do projeto acessíveis e a desvantagem desta plataforma é, entretanto, o custo elevado.

Visando o desenvolvimento de uma plataforma robótica para o ensino de controle, este trabalho tem como finalidade a construção de um robô equilibrista (pêndulo invertido Segway), como plataforma robótica aberta para o ensino de controle. Para tal, algumas premissas são estabelecidas, como: arquitetura aberta e acessível, desenvolvimento com softwares gratuitos, baixo custo (pela utilização de componentes eletrônicos acessíveis), fácil montagem (pelo uso de conectores e suportes simples), alta confiabilidade (a partir de estrutura mecânica rígida e dispositivos eletrônicos dimensionados), alta flexibilidade e adaptabilidade (permitindo utilização de diferentes técnicas de controle).

Diante das condições estabelecidas, propõe-se o desenvolvimento do robô empregando um sistema embarcado de tempo real (RTOS) sob microcontrolador ATmega328p (disponível na plataforma Arduino UNO), programado com o FreeRTOS, além de dois sensores-transdutores de medição inercial, circuito ponte $\mathrm{H}$ para controle de um par de motores $\mathrm{CC}$ e bateria de polímero de lítio de duas células, inclusive estrutura mecânica em fenolite e ABS, para fixação dos componentes.

Todo material desenvolvido encontra-se disponível de forma pública e gratuita nos repositórios do GitHub (https:// github.com/alexandremstf/opendulo) e Autodesk (https : //a360.co/2JfPLGa). E afim de validar a plataforma proposta assim como o funcionamento de todos os componentes do robô, utiliza-se o controlador PID, descrito na subseção 2.4. O algoritmo desenvolvido foi capaz de realizar o equilíbrio do robô. A estrutura do robô com seus componentes pode ser vista na Figura 1.

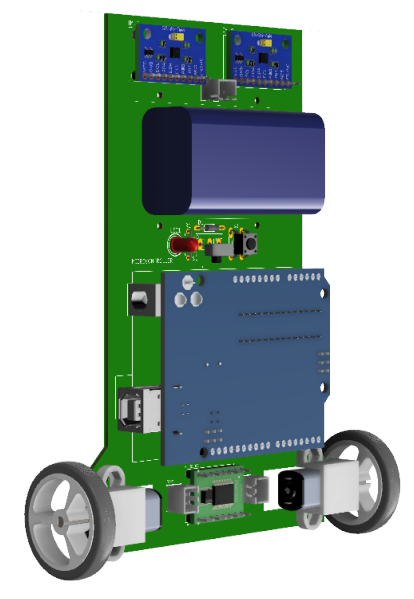

Figura 1. Representação do robô equilibrista como plataforma de ensino.

Este documento organiza-se como segue: na Seção 2 observa-se a fundamentação teórica de conteúdos relacionados ao desenvolvimento de pêndulos. A metodologia utilizada para o projeto do pêndulo, assim como os componentes que o compõem, são apresentados na Seção 3, enquanto experimentos e resultados são analisados e discutidos na Seção 4. Por fim, a Seção 5 apresenta as conclusões a respeito do trabalho produzido e aponta direções futuras para crescimento do projeto.

\section{FUNDAMENTAÇÃO TEÓRICA}

Sendo um problema recorrentemente abordado na literatura como não trivial, faz-se necessário para fundamentar o desenvolvimento da plataforma, um estudo aprofundado do comportamento e das características do robô. Nesta seção explicita-se a base teórica para tal problema.

\subsection{Modelo matemático}

O modelo matemático do problema abordado neste trabalho é o proposto por Howard and Bushnell (2015), construído a partir do modelo do motor e do modelo da estrutura, descritos por equações não-lineares. Combinando-os, pode-se linearizar o sistema em torno do seu ponto de equilíbrio (ângulo do pêndulo $\alpha=0$ [rad]). A representação mais direta do modelo, comumente aplicada para o controle, é a representação em espaços de estados, dada por:

$$
\begin{aligned}
& \dot{x}=A x+B u \\
& y=C x+D u
\end{aligned}
$$

Em que as variáveis de estado $x$ são definidas como $x=$ $[\alpha \dot{\alpha} X \dot{X}]^{\top}$, onde $\alpha$ representa o ângulo entre o pêndulo e o eixo vertical e $X$ o deslocamento horizontal. A entrada $u$ é a tensão

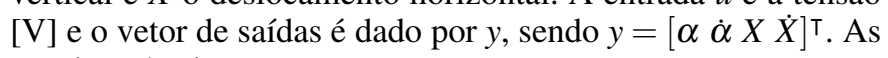
matrizes do sistema são: 


$$
\begin{aligned}
& A=\left[\begin{array}{cccc}
0 & 1 & 0 & 0 \\
\frac{g q_{1} q_{4}}{q_{1}^{2}+q_{2} q_{4}} & \frac{q_{3}\left(q_{1}-q_{4} r_{w}\right)}{\left(q_{1}^{2}+q_{2} q_{4}\right) r_{w}} & 0 & \frac{q_{3}\left(-q_{1}+q_{4} r_{w}\right)}{\left(q_{1}^{2}-q_{2} q_{4}\right) r_{w}^{2}} \\
0 & 0 & 0 & 1 \\
\frac{g q_{1}^{2}}{q_{1}^{2}+q_{2} q_{4}} & -\frac{q_{3}\left(q_{2}+q_{1} r_{w}\right)}{\left(q_{1}^{2}-q_{2} q_{4}\right) r_{w}} & 0 & \frac{q_{3}\left(q_{2}+q_{1} r_{w}\right)}{\left(q_{1}^{2}+q_{2} q_{4}\right) r_{w}^{2}}
\end{array}\right] \\
& B=\left[\begin{array}{c}
0 \\
\frac{k_{t}\left(q_{1}-q_{4} r_{w}\right)}{R\left(q_{1}^{2}-q_{2} q_{4}\right) r_{w}} \\
0 \\
\frac{k_{t}\left(-q_{2}+q_{1} r_{w}\right)}{R\left(q_{1}^{2}-q_{2} q_{4}\right) r_{w}}
\end{array}\right] \quad C=\left[\begin{array}{llll}
1 & 0 & 0 & 0 \\
0 & 1 & 0 & 0 \\
0 & 0 & 1 & 0 \\
0 & 0 & 0 & 1
\end{array}\right] D=0
\end{aligned}
$$

Em que:

$$
\begin{gathered}
q_{1}=L m_{p} \\
q_{2}=I_{p}+L^{2} m_{p} \\
q_{3}=\frac{k_{b} k_{t}}{R} \\
q_{4}=-m_{p}-m_{w}-\frac{i_{c m, w}}{r_{w}^{2}}
\end{gathered}
$$

Tabela 1. Parâmetros do robô

\begin{tabular}{lll}
\hline \multicolumn{1}{c}{ Símbolo } & \multicolumn{1}{c}{ Parâmetro } & \multicolumn{1}{c}{$\begin{array}{c}\text { Valor } \\
\text { [Unidade] }\end{array}$} \\
\hline$g$ & $\begin{array}{l}\text { Aceleração da gravidade } \\
\text { Distância entre o centro das duas rodas } \\
L\end{array}$ & $\begin{array}{ll}\mathrm{ms}^{-2} \\
\text { até o centro do pêndulo }\end{array}$ \\
$m_{p}$ & Massa do pêndulo & $\mathrm{cm}$ \\
$I_{p}$ & Momento de inércia no centro do pêndulo & $\mathrm{g}$ \\
$m_{w}$ & Massa da roda & $\mathrm{g}$ \\
$I_{c m, w}$ & Momento de inércia no centro de massa da roda & $\mathrm{kgm}$ \\
$r_{w}$ & Raio da roda & $\mathrm{cm}$ \\
$R$ & Resistência do motor & $\Omega$ \\
$k_{b}$ & Constante da força contra-eletromotriz do motor & $\mathrm{Vs} / \mathrm{rad}$ \\
$k_{t}$ & Constante de torque do motor & $\mathrm{Nm} / \mathrm{A}$ \\
\hline
\end{tabular}

A Tabela 1 e a Figura 2 apresentam a representação do pêndulo, bem como todas as constantes necessárias para o modelo.

Devido ao baixo custo dos componentes empregados, em virtude da proposta, ressalta-se que muitos parâmetros necessários para representar o sistema do pêndulo não estão disponíveis. Diante desta limitação, obteve-se as constantes necessárias para representação do modelo empiricamente, através de simulações com o software gratuito de modelagem Fusion360 e ensaios de bancada, como método da curva de reação, abordados na Subsubseção 3.1.1 e Subseção 2.3, respectivamente.

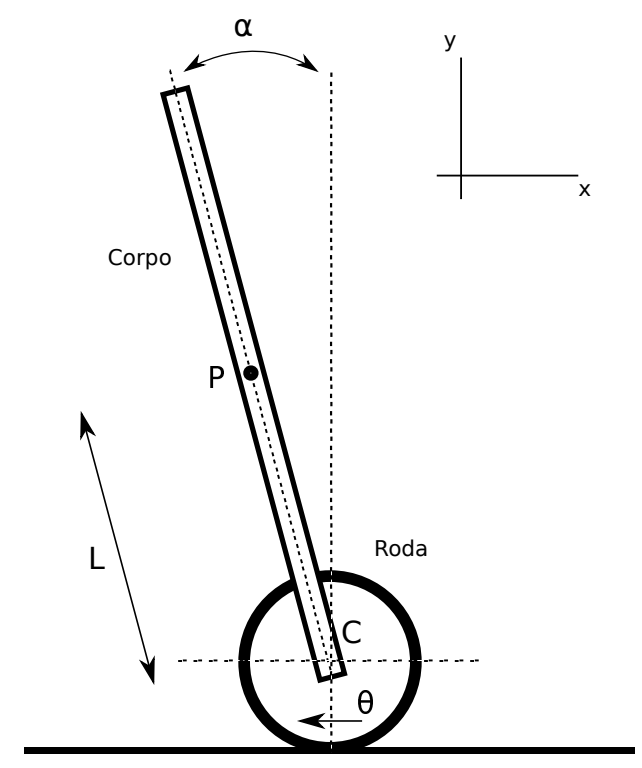

Figura 2. Representação do pêndulo invertido segway.

\subsection{Filtro complementar}

Filtros são ferramentas utilizadas para atenuar distúrbios e incertezas de um sistema. O filtro complementar apresenta-se como uma solução de fácil implementação para reduzir ruídos de sensores inerciais - como giroscópio e acelerômetro (Bueno and Romano, 2016). Um sistema típico, para sua aplicação, possui duas funções como entrada de dados, uma operando no domínio de alta frequência, sendo filtrada por um passaaltas, e a outra operando em baixa frequência, sendo filtrada por um passa-baixas. Pode-se unir as duas funções filtradas, já que ambas são complementares, formando uma reconstrução completa e sem ruídos do sinal (Pedley, 2013). A Figura 3 retrata como é utilizado o filtro complementar por uma unidade de medição inercial (IMU).

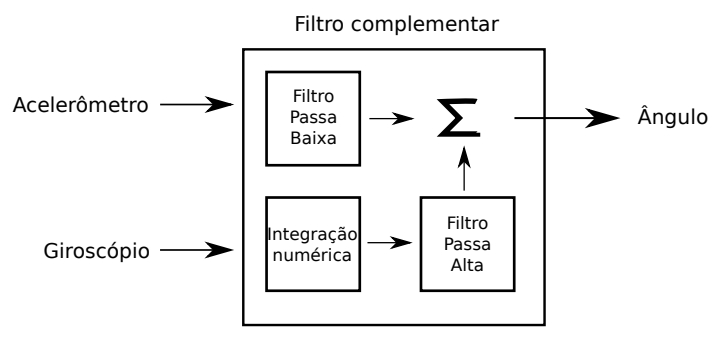

Figura 3. Representação do filtro complementar.

A utilização do filtro complementar permite melhor leitura de variáveis quando aplicado a projetos em que se utiliza a fusão de sensores para determinação de um mesmo parâmetro, como a posição angular.

\subsection{Método da curva de reação}

Cohen-Coon e Ziegler-Nichols propuseram regras para determinar os valores do ganho proporcional, do tempo integral e do tempo derivativo de um controlador PID. A proposta por Cohen-Coon melhora a sintonia do controlador levando a uma menor dependência em relação à constante de tempo $\tau$ e ao atraso L do sistema. Já a proposta por Ziegler-Nichols revela 
uma elevada sensibilidade de desempenho para diferentes valores da razão entre o atraso L e a constante de tempo $\tau$ do sistema (Torga, 2016).

A constante de tempo $\tau$ e o atraso L do sistema, podem ser obtidos, experimentalmente, por meio da curva de reação do processo em malha aberta, em resposta a uma entrada degrau (Ogata, 2009). Se o sistema não possuir polos complexos conjugados dominantes, então a curva de resposta será em forma de "S", de acordo com a Figura 4.

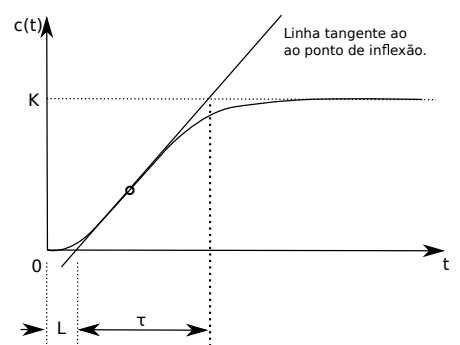

Figura 4. Curva de reação em função do tempo.

O tempo de atraso $(\mathrm{L})$ e a constante do tempo $(\tau)$ caracterizam a dinâmica do sistema e permitem sua representação simplificada pela função de transferência de primeira ordem $\left(G_{\mathrm{s}}\right)$, determinada pela Equação 7 .

$$
G_{\mathrm{s}}=\frac{K_{\mathrm{s}} e^{-L s}}{1+\tau s}
$$

Os parâmetros da Equação 7 são obtidos traçando-se uma reta tangente ao ponto de inflexão da curva "S" (ponto caracterizado por apresentar a derivada máxima em regime transiente) e estabelecendo os pontos de interseção da reta tangente com o eixo do tempo e a curva c(t) $=\mathrm{K}$, de acordo com a Figura 4 .

\subsection{Controle PID}

O controle proporcional-integrativo-derivativo (PID) fornece a solução mais simples e eficiente para vários problemas de controle do mundo real. Ambas as respostas, transiente e permanente, são atendidas com sua funcionalidade de três termos (ou seja, P, I e D). Desde a sua invenção, a popularidade do controle PID cresceu tremendamente. Os avanços na tecnologia digital tornaram o sistema de controle automático. Mesmo assim, o sistema de controle automático oferece um amplo espectro de opções para esquemas de controle e mais de $90 \%$ dos controladores industriais ainda são implementados com base nos algoritmos PID, particularmente no nível mais baixo, já que nenhum outro controlador pode corresponder à simplicidade, aplicabilidade e facilidade de uso oferecidas pelos controladores PID (Prasad et al., 2014).

Por este se apresentar como o método mais simples e eficiente de controle, com base em abordagens da literatura, foi o utilizado na validação da plataforma. Para estabilizar o pêndulo invertido na posição vertical usando a abordagem de controle PID, desenvolve-se o controlador de posição angular. Para tal, projeta-se o controlador determinando os parâmetros $\mathrm{K}_{p}, \mathrm{~K}_{i}$ e $\mathrm{K}_{d}$, segundo a lei de controle da Equação 8.

$$
U(s)=K_{p} E(s)+\frac{K_{i}}{s} E(s)+s K_{d} E(s)
$$

\subsection{Sistema Operacional de Tempo Real}

A aplicação do controle, bem como dos filtros, depende diretamente do período de amostragem e do período de atuação do controlador. Para o bom funcionamento de ambos é necessário garantir que o tempo de amostragem seja fixo, já que os ganhos do controlador, por exemplo, são determinados para um tempo de amostragem fixo e conhecido. Sendo assim, é imperativo para o funcionamento atender requisitos de tempo real. Portanto, um sistema operacional de tempo real pode ser aplicado, uma vez que é necessário garantir períodos determinados para a execução das tarefas de leitura e atuação do controle.

Um sistema operacional é um programa computacional composto de funções que abstraem o sistema de baixo nível e facilitam o uso de aplicações para os usuários. Um sistema operacional de tempo real, além de ter as características de um sistema operacional, deve também atender requisitos que garantam a execução de múltiplas tarefas simultaneamente e permita a execução dessas tarefas em um tempo determinado, de forma que prazos estabelecidos sejam atendidos ( $\mathrm{Li}$ and Yao, 2003).

\section{METODOLOGIA}

Esta seção destina-se à exploração dos elementos que compõem o projeto do pêndulo invertido. Faz-se o levantamento de custo do projeto, bem como justificativa da adoção de cada componente, observando sempre as premissas da proposta educacional. Por meio da revisão bibliográfica a literatura mostra a necessidade do conhecimento dos parâmetros mecânicos e eletrônicos do robô para a resolução do problema proposto, através das funções de transferências apresentadas anteriormente. Todavia, na prática, optou-se no projeto pelo menor custo possível, tornando inviável a obtenção dos parâmetros necessários através de datasheets. Métodos empíricos apresentados na literatura foram determinantes para obtenção de funções de transferências aproximadas. Utilizando software educacional gratuito de modelagem 3D, Fusion360, os parâmetros mecânicos foram simulados de maneira a auxiliar no projeto do controlador do pêndulo. Da mesma forma, para a aproximação do modelo do motor, o método da curva de reação foi essencial. Aliando esses métodos obteve-se de maneira satisfatória parâmetros para o controlador PID.

Além dos parâmetros obtidos, para a correta resposta do controlador, foi utilizado o filtro complementar, que reduziu ruídos e falhas das leituras dos sensores, gerando melhor resposta para a posição angular do pêndulo.

Integrando todos elementos, controlador e funções de transferências do sistema, componentes eletrônicos e mecânicos, por meio do sistema operacional de tempo real, foi possível validar a proposta da plataforma aberta para o projeto Open-dulo.

\subsection{Dispositivos de hardware}

Os componentes que compõe a parte física do pêndulo segway foram escolhidos considerando a minimização do custo e a facilidade de implementação. Desta forma, pôde-se elaborar uma plataforma financeiramente acessível e de baixa complexidade. A Tabela 2, a seguir, lista os componentes utilizados na montagem do pêndulo, assim como uma estimativa de custo. 
Tabela 2. Orçamento de componentes

\begin{tabular}{lcccc}
\hline Item & Fabricante & Qtd. & Código & Preço Total (R\$) \\
\hline Arduino UNO & Open-source & 1 & REV3 & 30,00 \\
IMU & Open-source & 2 & MPU9250 & 54,00 \\
Ponte H & Pololu & 1 & 713 & 20,00 \\
Micromotor & Pololu & 2 & 3063 & 160,00 \\
Rodas & Pololu & 2 & 1087 & 20,00 \\
Bateria & Turnigy & 1 & N460.2S.25 & 70,00 \\
Componentes & - & 1 & - & 36,00 \\
\hline Total & & & & 390,00 \\
\hline
\end{tabular}

Estrutura física Para produzir o pêndulo segway foi utilizada como estrutura mecânica a placa de circuito impresso de fenolite, a qual comporta todos os componentes do robô. Esta abordagem foi adotada para facilitar a conexão dos componentes elétricos e fixação dos componentes mecânicos. Como observado em outros trabalhos, através da revisão da literatura, atentou-se para a construção de um protótipo com maior confiabilidade, prevenindo desconexões elétricas (mau contato) e mecânicas, a partir de oscilações (vibrações) e do manuseio do robô.

Componentes eletrônicos Esta subseção enumera, descrevendo brevemente, os dispositivos utilizados neste projeto.

(1) Microcontrolador - ATmega328p

O microcontrolador adotado para este projeto é o ATmega328p, como observado na Figura 5. Este chip está disponível na plataforma Arduino UNO e, por apresentar vasto material de apoio, baixo custo e suportar o FreeRTOS, este dispositivo atende aos requisitos do projeto. As funcionalidades apresentadas por esta microcontroller unit (MCU) e necessárias ao projeto incluem:

- Timers - utilizados para gerenciamento das threads do sistema operacional e acionamento dos motores, via PWM;

- $\mathrm{I}^{2} \mathrm{C}$ - protocolo de comunicação dos sensores MPU9250;

- Saídas digitais - para controle do driver ponte H.

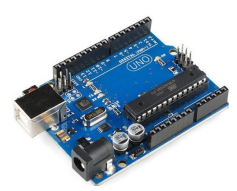

Figura 5. Arduino UNO REV3 - Atmega328p.

(2) IMU - MPU9250

A fim de fazer a leitura da posição angular do robô pêndulo, faz-se necessário a utilização de um dispositivo sensor. Para esta plataforma optou-se pela utilização de duas unidades MPU9250, ilustrada pela Figura 6. Contando com 9 graus de liberdade (3 de acelerômetro, 3 de giroscópio e 3 de magnetômetro), cada um destes sensores permite a leitura, em três direções, de aceleração, velocidade angular e campo magnético, respectivamente. Este dispositivo comunica-se com o microcontrolador pelo protocolo $\mathrm{I}^{2} \mathrm{C}$.

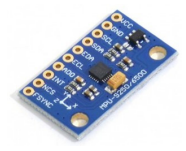

Figura 6. IMU - MPU9250.

\section{(3) Ponte H - TB6612FNG}

Para acionamento dos motores e rotação em ambos sentidos faz-se necessário a utilização de um circuito chopper de 4 quadrantes - neste caso, uma ponte $\mathrm{H}$. O circuito integrado TB6612FNG, da Figura 7, é construído com MOSFETs, possui diodos de roda livre, controle de sentido de rotação e habilitação do chip, além da capacidade de acionar dois motores individualmente.

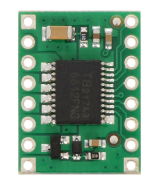

Figura 7. Ponte H - TB6612FNG.

\section{(4) Micromotor CC}

A fim de garantir locomoção ao robô pêndulo invertido, são utilizados dois micro motores CC Pololu com caixa de redução de 51,45:1 e rotação a vazio, sob tensão nominal de 6 [V], de 590 [rpm] no eixo acoplado à roda, de 32 [mm] de diâmetro. O conjunto roda-motor pode ser observado na Figura 8.

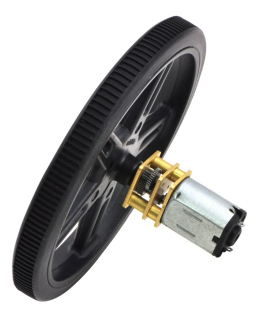

Figura 8. Micromotor CC

(5) Bateria Li-poly

De forma a alimentar todos os dispositivos eletrônicos do pêndulo, utiliza-se uma bateria de polímero de lítio de 7,4 [V], 1000 [mAh] e 65 [g], ilustrada na Figura 9.

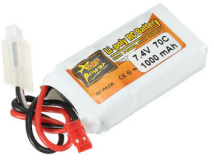

Figura 9. Bateria LiPoly 2S.

\subsection{Elementos de software}

Especifica-se aqui os componentes computacionais envolvidos na elaboração do projeto da plataforma educacional do pêndulo invertido. Para esta etapa considerou-se principalmente a facilidade de implementação.

FreeRTOS O FreeRTOS é um Sistema Operacional de Tempo Real destinado a aplicações de sistemas embarcados. É open source e possui todas as funções de um Sistema Operacional de Tempo Real. Neste trabalho são utilizados alguns recursos do FreeRTOS como a aplicação de duas tarefas, uma de leitura dos sensores e uma para a atuação do controle. Outro recurso é a utilização de um semáforo binário que protege os dados medidos pelos sensores do robô. Adicionalmente, o FreeRTOS 
garante períodos fixos e determinados para atuação das tarefas de controle e dos filtros, gerenciando eventos cíclicos.

\section{ANÁLISE E RESULTADOS}

Após a implementação do sistema desenvolvido, conseguiu-se ajustar os parâmetros do controlador e garantir o equilíbrio do robô. A partir de ensaios, pôde-se testar todos os componentes eletrônicos, assim como o código, que realizou com sucesso a leitura dos sensores, através do barramento $\mathrm{I}^{2} \mathrm{C}$, e também configurou corretamente todos os registradores necessários para o correto funcionamento do sistema.

Validou-se a proposta do kit desenvolvido, capaz de atender aos requisitos estabelecidos, e também a configuração das tarefas criadas no sistema operacional de tempo real, destinadas ao controle e a leitura dos sensores com deadlines fixos. Esta confirmação foi possível através de testes de depuração que demonstraram o acesso às tarefas de acordo com prioridades, bem como sua execução em tempos cíclicos e bem definidos.

Conseguiu-se, como planejado, a elaboração de uma plataforma educacional de baixo custo, apresentada na Figura 10, em que é possível desenvolver e implementar diferentes técnicas de controle.

Observou-se, por fim, que todos os componentes e a estrutura utilizada no desenvolvimento deste protótipo são suficientes para utilização em ambientes acadêmicos, como ferramenta de ensino.

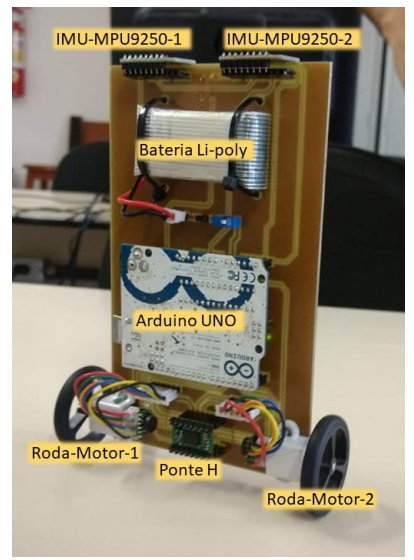

Figura 10. Plataforma Open-dulo com seus principais componentes indicados e se equilibrando por meio do controle PID.

\section{CONCLUSÃO E TRABALHOS FUTUROS}

Este trabalho propôs a construção de uma plataforma educacional de robótica, baseado no pêndulo invertido, com a implementação de controle de posição angular em linguagem $\mathrm{C}++$ de programação e uso do sistema de tempo real FreeRTOS.

A partir dos componentes especificados, abordagem para concepção do pêndulo, linguagem de programação e sistema operacional adotados, o robô desenvolvido teve como princípio atender aos requisitos necessários de uma plataforma aberta, para ensino de controle. Atentou-se, neste trabalho, ao custo de desenvolvimento, facilidade de manuseio do robô, confiabilidade das conexões, adaptabilidade e maleabilidade da plataforma.
A fim de incrementar funcionalidades à plataforma, sugere-se modificações e novas funções para trabalhos futuros, como:

- Revisão da modelagem do pêndulo segway;

- Implementação de sensor de velocidade para os motores;

- Implementação de comunicação sem fio, para armazenamento de dados e controle remoto do robô equilibrista;

- Implementação de análises gráficas de regime transiente e rejeição de distúrbios;

- Implementação de controle de posição angular e de controle de posição angular e linear (separadamente), por diferentes métodos, como PID, LQR e Fuzzy;

- Implementação de demais filtros, como Kalman, Complementar e de Partículas;

- Adaptação para utilização de diferentes inertial measurement units (IMU).

Devido ao caráter open-source do projeto é esperado que outros pesquisadores e alunos possam implementar este robô e participar ativamente do repositório no GitHub. Dessa forma, estas sugestões e outros avanços estarão disponíveis para a comunidade acadêmica e podem apoiar o ensino/aprendizado de técnicas de controle, eletrônica e programação.

\section{REFERÊNCIAS}

Bueno, A.G. and Romano, R.A. (2016). Filtro complementar aplicado a medida de inclinação de plataformas móveis.

Howard, B. and Bushnell, L. (2015). Enhancing linear system theory curriculum with an inverted pendulum robot. In 2015 American Control Conference (ACC), 2185-2192. IEEE.

Jamieson, P. and Herdtner, J. (2015). More missing the boat - arduino, raspberry pi, and small prototyping boards and engineering education needs them. In 2015 IEEE Frontiers in Education Conference (FIE), 1-6. doi:10.1109/FIE.2015. 7344259.

Li, Q. and Yao, C. (2003). Real-time concepts for embedded systems. CRC Press.

Nawawi, S., Ahmad, M., and Osman, J. (2008). Real-time control of a two-wheeled inverted pendulum mobile robot. World Academy of Science, Engineering and Technology, 39, 214-220.

Ogata, K. (2009). Modern control engineering. Prentice Hall Upper Saddle River, NJ.

Pedley, M. (2013). Tilt sensing using a three-axis accelerometer. Freescale semiconductor application note, 1, 20122013.

Prasad, L.B., Tyagi, B., and Gupta, H.O. (2014). Optimal control of nonlinear inverted pendulum system using pid controller and lqr: performance analysis without and with disturbance input. International Journal of Automation and Computing, 11(6), 661-670.

Soriano, A., Marin, L., Valles, M., Valera, A., and Albertos, P. (2014). Low cost platform for automatic control education based on open hardware. IFAC Proceedings Volumes, 47(3), 9044-9050.

Torga, D.S. (2016). Desenvolvimento de uma plataforma didática para práticas de controle de velocidade de motor de corrente continua.

Tuomi, I. (2013). Open educational resources and the transformation of education. European Journal of Education, 48(1), $58-78$.

Vega, J. and Cañas, J. (2018). Pibot: An open low-cost robotic platform with camera for stem education. Electronics, 7(12), 430. 